\title{
Cultural competence of nurses in Pudong New Area, Shanghai: a mixed-method study
}

Original article

Li-Li Maa, Hui Jiang ${ }^{\mathrm{b}, *}$, You-Qing Peng ${ }^{\mathrm{a}, *}$

Received: 29 May 2019; Accepted: 13 August 2019; Published: 20 June 2020

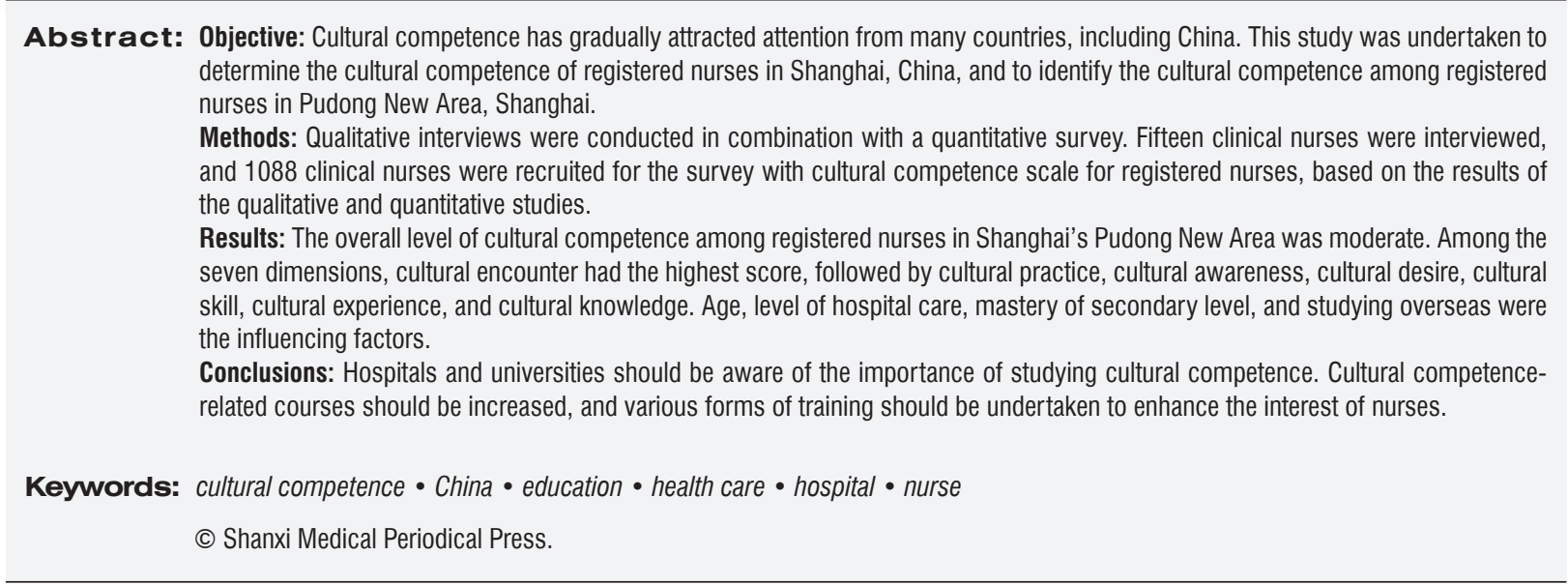

\section{Introduction}

Since the 20th century, cultural disparities in the healthcare system have initiated health services research, system reform, and changes in policy. ${ }^{1}$ The US Institute of Medicine gradually focused on this area and recommended that transcultural education should be combined with the training of health professionals. Actually, cultural competence has begun to appear as a strategy to address health disparities..$^{2-4}$ Since China joined the World Trade Organization (WTO), people with diverse cultural backgrounds have flocked to China. China is rapidly becoming the nation with the greatest racial and ethnical diversities in the world. The diversity represents a challenge to health-care providers. It is apparent that cultural tension and misunderstanding between

How to cite this article: Ma LL, Jiang H, Peng YQ. Cultural competence of nurses in Pudong New Area, Shanghai: a mixed-method study. Front Nurs. 2020; 2: 119-128.

* Corresponding authors.

E-mail: jianghuitest@163.com (H. Jiang); 2856580686@qq.com (Y. -Q.Peng). 
patients and health-care providers would increase patient mistrust and dissatisfaction, as well as causing decreased confidence in the medical system and poor health outcomes. ${ }^{5-7}$ Enhancing greater cultural competence among health-care providers is becoming an increasingly crucial goal. There have been few studies relating to cultural competence among registered nurses in Shanghai. Therefore, this study has been undertaken to determine the cultural competence of registered nurses in Shanghai, China.

\subsection{Definition of cultural competence}

Nowadays, cultural competence has become a prerequisite for health-care professionals working in transcultural societies. Cultural competence in the health-care system describes the ability to provide care to patients with diverse values, beliefs, and behaviors, including the adjusting of care delivery to meet their social, cultural, and linguistic needs. ${ }^{8,9}$ Cultural competence, which originated in the US, initially focused on teaching the beliefs and characteristics of specific cultural and ethnic groups. Cultural competence has been defined in various ways, but it is usually comprehended as the attitudes, knowledge, and skills necessary for providing quality care to a diverse population (i.e., the capacity to deliver culturally appropriate care). ${ }^{10}$ The concept of cultural competence switches to the topics related to ethnic inequalities in the quality of health care. ${ }^{11,12}$ In this article, cultural competence is defined as follows: knowledge, skills, awareness, experience, practice, desire, and encounter, which are essential for healthcare providers to provide acceptable cultural care. Competence is an ongoing process that involves accepting and respecting differences. ${ }^{13}$ According to Jeffreys, ${ }^{14}$ the need for culturally competent care among healthcare professionals is described in many reports in literature from Australia, Canada, Israel, Sweden, South Africa, and Great Britain. There is a vast amount of international literature on cultural competence. A study was done to assess the cultural competence of medical students and physicians to identify the gaps in knowledge and culturally competent behavior. ${ }^{15}$ Another survey was distributed to compare and analyze cultural competence in beginning and graduating nursing students. ${ }^{16}$ A study was also conducted in Switzerland to compare cross-cultural skills between physicians and nurses. Cultural competence has gained importance worldwide. ${ }^{1}$

\subsection{Cultural competence in China}

Several studies have also been conducted to evaluate cultural competence among nurses or nursing students in China. ${ }^{17}$ Nurse educators or nurse administrators have gradually realized the importance of competence among nurses. China is a developing country and has carried out the policy of "reform and opening up" since the 1980s. As a result, China develops as does the whole education system. Currently, the nursing education system has been well established in China, including diploma, associate degree, baccalaureate, master's, and doctoral degree programs. ${ }^{18} \mathrm{~A}$ hierarchy of five levels of nurses, i.e., junior nurse, senior nurse, nurse in charge, assistant chief senior nurse, and chief senior nurse, is designed, with number of credits allotted in accordance with their educational background, ability in nursing service, and experience in nursing practice. ${ }^{19,20}$ There are three levels of hospitals (community hospitals, secondary-level hospitals, and tertiary-level hospitals) in China, which are categorized according to the functions and resources within the health-care system. According to the Statistics of China, there were 5271 community hospitals, 6472 secondary-level hospitals, and 1284 tertiary-level hospitals in China at the end of $2010 .{ }^{21}$ To meet the growing health-care demands of the large population, it was apparent that more nurses were required, especially nurses working in tertiary hospitals. The major nursing work forces comprised junior and associate degree nurses in Shanghai. The standards for nursing recruitment in tertiary hospitals are an associate degree or above. The level of English mastery was another concern for recruitment. Generally, students who received a baccalaureate degree past College English Test 4 or 6 (the higher the grade, the higher is the level of English mastery) were acceptable. More training courses related to cultural competence were arranged in tertiary hospitals. Nurses who worked in tertiary hospitals would have more opportunities to provide services for patients hailing from all parts of the world. Patients from different parts of the world have different cultural backgrounds; even in Shanghai, patients from different districts also have their own dialects, habits, and customs, which exhibit a number of cultural differences. To alleviate problems caused by cultural disparities, cultural competence is also attracting the attention of nurse educators or nurse administrators.

\subsection{Aims}

The purpose of the study was to identify the cultural competence of registered nurses in Pudong New Area, Shanghai, and to take measures to improve nurses' cultural competence and decrease nurses' cultural intolerance. The measures will eventually be conductive to eliminating the current nursing conflicts and strengthening the quality of nursing care. 


\section{Methods}

\subsection{Study process}

Qualitative interviews were combined with a quantitative cross-sectional survey in this study. The following two tools were used: (1) semistructured interviews and (2) structured survey. Collection of data was based on literature review and surveys in support of the study objectives. ${ }^{22,23}$ The data included demographics, hospital level, job position, educational level, years of working experience, level of English mastery, overseas studying/ working experience, and experience of attending multicultural nursing-related training courses. In the interviews, the questions asked were as follows: (1) What are your perceptions regarding cultural competence? (2) Do you think a cultural competence course is essential for nurses? Why? (3) What are the major barriers for nurses in applying cultural competence in clinical practice? (4) What would be helpful to increase cultural competence among nurses? (5) What kind of training would increase the cultural competence of nurses? (6) What are the major factors influencing cultural competence among nurses? Cultural competence among nurses was also evaluated using a selfdesigned questionnaire that included 7 subscales and 84 items. The survey questionnaire was developed by the research team, based on a careful literature review and the results of qualitative interviews. The CampinhaBacote Inventory for Assessing the Process of Cultural Competence-Revised (IAPCC-R) was initially chosen for the survey; however, there were significant cultural differences between China and other countries. If the IAPCC-R was used directly, there would be many misunderstandings affecting the survey results and the true results cannot be presented. Thus, a self-devised survey questionnaire was constructed based on the IAPCC-R, the Transcultural Nursing Theory, evidencebased practice theory, Chinese culture and national conditions, and Delphi results. The survey participants were asked to rate their perception of cultural competence on a five-point Likert scale, ranging from " 5 " (strongly agree) to "1" (strongly disagree). The higher the score, the more favorable is the perception that the participant has toward cultural competence. The questionnaire was tested for content-related validity and content validity, which were reviewed by 18 experts in the areas of nursing education, nursing management, and nursing researchers. The content validity was 0.913 . Cronbach's alpha was 0.978 . A pilot study involving 30 nurses was conducted to test the clarity and applicability of the tool. The reliability and validity of the instrument were at acceptable levels.

\subsection{Sample and setting}

The study was conducted in 23 community hospitals, 8 secondary hospitals, and 2 tertiary hospitals in Pudong New Area, Shanghai. There were 15 clinical nurse interviews, and 1088 clinical nurses were recruited for the survey with the convenience sampling method. The participants were chosen based on the following criteria: (1) full-time registered nurses working in clinical departments; (2) worked in a clinical department for at least 1 year; (3) willing to participate in the survey; and (4) could give informed consent. Fifteen nurses were interviewed, and 1088 nurses participated in the survey.

\subsection{Data collection and analysis}

Data were collected between October 2015 and January 2016. The interviews were held in a separate quiet room, and each interview lasted for at least $30 \mathrm{~min}$. All of the in-depth interviews were tape-recorded after getting the consent from the participants. The interviews were converted into texts within $48 \mathrm{~h}$ of completion. The questionnaires were manually distributed to potential respondents by the researchers. Each questionnaire required 10-15 min to complete. To protect the privacy of the participants and to increase the response rate, the participants were asked to put the completed questionnaires into envelopes and then place them in a sealed box in the unit. Subsequently, the head nurse returned the sealed questionnaires to the investigator. All of the nurses in the selected wards were recruited. Double entry of the survey data into a password-protected computer program was then completed, and the data were checked for consistency and accuracy. Statistical Package for Social Sciences software for Windows version 18.0 (SPSS, Inc., Chicago, IL, USA) was used to analyze the data. Descriptive statistics (means and standard deviations) were calculated to summarize the data. The mean and standard deviation were used to show the perception of each item as given by the respondents. The relationship between the cultural competence among registered nurses and demographic variables was analyzed with analysis of variance (ANOVA). A $P$-value $<0.05$ (twosided) was considered as statistically significant.

\section{Results}

\subsection{Demographics}

All 15 nurses who participated in the qualitative interviews were females (age range: 21-36 years; mean age: $28.14 \pm 2.81$ years), and their hospital working experience ranged from 1 year to 16 years. Thirteen 
nurses were married (86.67\%). Four nurses had an associate degree and 11 nurses had a baccalaureate degree. Eight nurses were from tertiary hospitals, four nurses were from secondary hospitals, and three nurses were from community hospitals. There were two nurses from the intensive care unit (ICU), two nurses were from the Cardiovascular Ward, two nurses were from the Nephrology Ward, two nurses were from the Anorectal Surgery Ward, two nurses were from the Emergency Department, two nurses were from wards involving overseas patients, and three nurses were from the Outpatient Department. Four nurses had overseas study/training experience and seven nurses had joined multicultural nursing courses. A total of 1088 registered nurses participated in the quantitative survey. Seven registered nurses were male $(0.6 \%)$ and 1081 registered nurses were female $(99.4 \%)$; the age range was 21-55 years (mean age: $29.6 \pm 6.9$ years), and the hospital working experience ranged from 1 year to 36 years. Furthermore, 66 registered nurses $(6.1 \%)$ had diploma degrees, 537 (49.5\%) had associate degrees, and 481 $(44.4 \%)$ had baccalaureate or higher degrees.

\subsection{Focused interview findings}

Two themes with several subthemes emerged from the data in relation to the perception of cultural competence among nurses. All of the interviewees had positive attitudes toward cultural competence, but the understanding of cultural competence was not all-inclusive. The interviewees thought cultural competence was important for all nurses, and training should be undertaken to broaden the scope of knowledge among nurses.

\subsubsection{Theme 1: Perceptions of cultural competence among nurses}

All of the interviewed nurses were willing to obtain more knowledge and skills to increase their cultural competence.

\subsection{1. Subtheme I: Nurses considered that cultural competence is important for quality nursing care}

Nurses have gradually become familiar with the concept of cultural competence. Nurses thought that the concept of cultural competence could deepen the connotation of quality nursing care.

"I think, nowadays, China has implemented the policy of quality nursing care, so there are more requirements for nurses. To be qualified as a registered nurse or a good primary nurse, I think pure nursing knowledge and practical skills are not sufficient. More emphasis was put on the humanistic care, so I think cultural competence is very important for nurses, especially for quality nursing care."

"Hospitals in Shanghai take various measures to improve nursing quality, which includes software and hardware, so I think the perception and application of cultural competence increases nursing quality."

\subsubsection{Subtheme II: Nurses considered that cultural competence reflects comprehensive nursing ability}

Now, the understanding regarding the ability of nurses has been updated. Nurses should be proficient in knowledge and skills to be an excellent nurse.

"I think the ability of a nurse includes nursing knowledge, nursing practical skills, humanistic care, communicating skills, cultural sensitivity, cultural consciousness, and scientific research ability."

"Nowadays, nurses meet various patients. Nurses should have a broad scope of knowledge and can cope with different situations."

"I think cultural competence is an ability which can help nurses fit various environments. If we work in the Emergency Department, we should know how to manage emergent cases, and how to communicate with patients and their relatives in special emergent situations. If we work in the wards involving overseas patients, we should know more about patient taboos, diets, insurance payment, and cultural knowledge. We also should have scientific sensitivities and use the scientific method to solve clinical problems."

\subsection{3. Subtheme III: Nurses considered that cultural competence could increase communication skills}

Communication skills are important for nurses because communication can ease misunderstanding and increase trust between nurses and patients.

"I think through cultural competence training, I can know what we could learn to improve our quality of service and how to communicate with patients. Cultural competence is the process of accumulation. The more practice in communicating with patients with cultural competence consciousness, the more experience in cultural competence that is acquired. Then, the entire level of communication skills is increased."

"There is an abundance of hospital violence occurring in China, and the reason can be ascribed to a lack of trust and communication. Cultural competence can shorten the distance between nurses and patients."

"There was much news reported in the newspapers and on the Internet. Of course, some conflicts were derived from unacceptable medical results. But more 
problems come from the lack of effective coping, especially among junior nurses. Last time, my colleague got a complaint from a patient just because of linguistic misunderstanding between the native Shanghai patient and the junior nurse from Anhui Province. So, if the nurses were trained and knew more about linguistic offense and how to compensate for what they say, then lots of complaints can be decreased."

\subsubsection{Theme 2: Perceptions among nurses on how to increase the cultural competence of nurses}

When nurses were interviewed for what methods should be taken to increase nurses' cultural competence, they all mentioned culture-related courses, including language courses, courses relating to communication skills, practical skills of cultural competence, knowledge about religion and customs, privacy protection, and so on. Many nurses mentioned that they wanted to have the chance to join the foreign ward or probate at the foreign ward. Some mentioned that multicultural training could be added to the nurses' annual courses or be a part of new employers' training courses.

\subsubsection{Subtheme I: Nurses thought that language was the major barrier for the practice of cultural competence}

Three nurses referred to language as a major barrier for the practice of cultural competence in daily work.

"As a clinical nurse, I think language, not only are English, French, and Japanese major barriers for me, but some Chinese regional dialects, such as Fujian and Wenzhou dialects, are also really difficult for me to understand. When I care for a patient from Wenzhou, I do not know what the patient wants, and the only way I communicate with the patient is by body language, script, or translation by a relative. I cannot educate the patient or communicate with the patient directly".

"There are many local dialects in China, even in Shanghai, 4-5 Shanghai dialects can be heard. It is said that Shanghai dialects are trained in some hospitals, so languages are important for increasing cultural competence."

"Of course, language is a barrier for us. Foreign language is only a part of the problem, because most of our junior nurses have bachelor degrees; the major barrier for English is speaking."

\subsubsection{Subtheme II: Nurses thought that cultural competence should have more content}

Nurses also mentioned cultural competence course content and course arrangements in the interviews.
"When we discussed cultural competence, the main perception was languages, customs, religions, and personal preferences. I thought cultural competence had more meaning, such as insurance, knowledge about traditional Chinese medicine (TCM), and even some superstition. We should eradicate the rigid perception about cultural competence by updating information and conception gradually and repeatedly.

Nurse involved in caring for overseas patients said, "My suggestion is that we can set admission criteria relating to cultural competence for new nurses. Then, nurses will know and apply cultural competence into daily work."

"The major barrier for my nursing skills is the lack of some basic knowledge. Because patients always think nurses know everything, when they need information and help, nurses are the first person who can help them. Sometimes, they will ask me about their medical insurance, traffic information, medical records copy procedures, and hospital regulations, which are not related to our nursing procedures. So, I think I need more information and knowledge, which can be accumulated with our experience."

\subsubsection{Subtheme III: Nurses suggested that more forms of training courses should be undertaken}

Nurses had many ideas about the training schemes. Large-class instruction is the least favorite training form for nurses.

"The major training forms were classes, media presentations, and seminars. My advice is to set up some scene simulation, such as fire-fighting exercises, shopping simulation, case discussion, and brain storming. More methods should be taken to increase the interest of nurses, and nurses can actively join the study. I also want the hospital to give us opportunities to study outside and to know more about new findings and updated information."

"I want to choose the courses in which I am interested. We should not be forced to attend all the courses. Case study is really attractive for me."

"Nurses with different work experiences and diplomas should have different training courses. The form of the mutual help group can be used for junior and senior nurses. The spirit of competitiveness can also be induced in the training. Exams are not always effective for absorbing the cores of cultural competence."

"I think much training can be practiced during morning conference and night conference, training should not be limited to only formal classes. During morning conference, after the night-shift nurse's reports, the head nurse and senior nurses can give more details about 
what our nurses should pay attention to. This kind of training is something like case study, which is more effective than ordinary class training."

\subsection{Questionnaire survey findings}

The survey showed that the current status of competence among registered nurses in Shanghai's Pudong New Area is of a moderate level (4.06 \pm 0.817$)$; among seven dimensions, the highest score was for cultural encounters $(4.21 \pm 0.614)$ and the lowest score was for cultural knowledge $(3.81 \pm 1.128)$ (Table 1). Oneway ANOVA was used to compare the average scores among the different variables. Among the variables, age was related to the cultural awareness of nurses, and those $>40$ years of age tended to have the highest level of cultural awareness. Job position was related to cultural encounters, and department head nurses tended to have the highest level of cultural awareness. The hospital level was related to cultural awareness, cultural knowledge, cultural skill, and cultural desire. Nurses in tertiary hospitals tended to have the highest level of cultural awareness and cultural desire. Nurses in secondary hospitals tended to have the highest level of cultural knowledge and cultural skill. Mastery of English in nurses was related to the level of cultural awareness and cultural desire. Learning a secondary foreign language and overseas studying/training experience were related to the level of cultural awareness (Table 2).

\section{Discussion}

\subsection{The necessity of studying nurses' cultural competence}

There are several research works related to cultural competence, ${ }^{24-27}$ but these studies have mainly focused on nursing students and no reports are available about the general cultural competence of Shanghai's registered nurses. And about the rating scales, the registered

\begin{tabular}{lccc}
\hline Dimensions & Items & Average scores & Ranges \\
\hline \hline Cultural encounter & 10 & $4.21 \pm 0.614$ & $1-5$ \\
Cultural practice & 6 & $4.18 \pm 0.625$ & $1-5$ \\
Cultural awareness & 16 & $4.13 \pm 0.887$ & $1-5$ \\
Cultural desire & 14 & $4.06 \pm 0.830$ & $1-5$ \\
Cultural skill & 11 & $4.04 \pm 0.796$ & $1-5$ \\
Cultural experience & 12 & $4.01 \pm 0.837$ & $1-5$ \\
Cultural knowledge & 15 & $3.81 \pm 1.128$ & $1-5$ \\
Total & 84 & $4.06 \pm 0.817$ & $1-5$ \\
\hline
\end{tabular}

Table 1. Cultural competence scores among registered nurses $(\mathrm{M} \pm \mathrm{SD})$. nurses' cultural competence scale was constructed based on the IAPCC-R, the Transcultural Nursing Theory, evidence-based practice theory, Chinese culture and national conditions, and Delphi results. Through qualitative and quantitative studies, we could well evaluate nurses' cultural competence in Shanghai, China. Furthermore, we could give suggestions to increase nurses' cultural competence through data analysis.

\subsection{Overall scores and scores for the seven dimension of cultural competences among nurses in Shanghai's Pudong New Area}

Our study showed that the overall level of cultural competence among registered nurses in Shanghai's Pudong New Area was $4.06 \pm 0.614$. A five-point Likert scale was used as the ranking tool, wherein three points indicated an average level, four points indicated moderate level, and five points indicated good level of competence. Therefore, according to the scores, the overall cultural competence of the registered nurses in Pudong New Area was of the moderate level; also, cultural encounters had the highest score, and cultural knowledge had the lowest score among the seven dimensions for registered nurses in Pudong New Area (Table 1).

\subsubsection{Overall cultural competence was at a moderate level}

The survey showed that the current status of cultural competence among registered nurses in Shanghai's Pudong New Area was at a moderate level (4.06 \pm 0.817$)$. The results may be related to the fact that the educational level of the registered nurses was relatively high in Pudong New Area (93.9\% of registered nurses had an associate or baccalaureate degree). Forty-six nurses $(4.23 \%)$ had overseas studying/training experience, and 73 nurses $(7.17 \%)$ had learned or had been learning a secondary foreign language. Most of the participants $(79.23 \%)$ were from secondary and tertiary hospitals, and large hospitals - which were boosted not only in numbers, but also in complexity - tended to have more patients. Apart from these reasons, Pudong New Area is located in Shanghai with the historical culture of opening to the outside, and it is also an economical center in China.

\subsubsection{Cultural encounter had the highest score}

Cultural encounter runs though all nursing procedures and daily communication. In nursing practice, nurses knew what to discuss with patients in advance, what should be explained, and what to remind the patients 


\begin{tabular}{|c|c|c|c|c|c|c|c|}
\hline Variables (n) & $\begin{array}{c}\text { Cultural } \\
\text { awareness }\end{array}$ & $\begin{array}{c}\text { Cultural } \\
\text { knowledge }\end{array}$ & $\begin{array}{l}\text { Cultural } \\
\text { skill }\end{array}$ & $\begin{array}{c}\text { Cultural } \\
\text { encounter }\end{array}$ & $\begin{array}{l}\text { Cultural } \\
\text { desire }\end{array}$ & $\begin{array}{c}\text { Cultural } \\
\text { experience }\end{array}$ & $\begin{array}{l}\text { Cultural } \\
\text { practice }\end{array}$ \\
\hline \multicolumn{8}{|l|}{ Age, years } \\
\hline$\leq 25(332)$ & $4.117 \pm 0.439$ & $3.832 \pm 0.640$ & $4.004 \pm 0.558$ & $4.174 \pm 0.470$ & $4.027 \pm 0.586$ & $3.999 \pm 0.524$ & $4.174 \pm 0.486$ \\
\hline 26-30 (303) & $4.108 \pm 0.460$ & $3.801 \pm 0.611$ & $3.995 \pm 0.500$ & $4.188 \pm 0.488$ & $4.042 \pm 0.525$ & $4.027 \pm 0.515$ & $4.174 \pm 0.528$ \\
\hline $31-40(319)$ & $4.095 \pm 0.477$ & $3.749 \pm 0.593$ & $3.970 \pm 0.480$ & $4.176 \pm 0.463$ & $4.003 \pm 0.547$ & $3.985 \pm 0.596$ & $4.165 \pm 0.582$ \\
\hline$\geq 40$ (128) & $4.270 \pm 0.574$ & $3.891 \pm 0.671$ & $4.088 \pm 0.567$ & $4.275 \pm 0.438$ & $4.113 \pm 0.522$ & $4.095 \pm 0.526$ & $4.277 \pm 0.471$ \\
\hline$F$ & 4.55 & 1.87 & 1.59 & 1.62 & 1.25 & 1.40 & 1.56 \\
\hline$P$ & $<0.05^{\star}$ & $>0.05$ & $>0.05$ & $>0.05$ & $>0.05$ & $>0.05$ & $>0.05$ \\
\hline \multicolumn{8}{|c|}{ Level of hospital care } \\
\hline $\begin{array}{l}\text { Community } \\
\text { hospital (222) }\end{array}$ & $4.002 \pm 0.499$ & $3.572 \pm 0.549$ & $3.842 \pm 0.471$ & $4.152 \pm 0.445$ & $3.892 \pm 0.522$ & $3.886 \pm 0.552$ & $4.104 \pm 0.528$ \\
\hline $\begin{array}{l}\text { Secondary } \\
\text { hospital (26) }\end{array}$ & $4.208 \pm 0.537$ & $3.901 \pm 0.561$ & $4.077 \pm 0.539$ & $4.161 \pm 0.590$ & $4.125 \pm 0.643$ & $4.094 \pm 0.515$ & $4.207 \pm 0.566$ \\
\hline $\begin{array}{l}\text { Tertiary hospital } \\
\text { (634) }\end{array}$ & $4.214 \pm 0.508$ & $3.825 \pm 0.612$ & $4.009 \pm 0.506$ & $4.198 \pm 0.455$ & $4.056 \pm 0.542$ & $4.022 \pm 0.511$ & $4.213 \pm 0.493$ \\
\hline$F$ & 7.86 & 18.66 & 13.19 & 1.21 & 6.96 & 7.07 & 2.58 \\
\hline$P$ & $<0.05^{\star}$ & $<0.05^{\star}$ & $<0.05^{\star}$ & $>0.05$ & $<0.05^{\star}$ & $>0.05$ & $>0.05$ \\
\hline \multicolumn{8}{|c|}{ Level of English mastery } \\
\hline $\begin{array}{l}\text { College English } \\
\leq \text { Level } 3 \text { (749) }\end{array}$ & $4.120 \pm 0.473$ & $3.798 \pm 0.624$ & $3.988 \pm 0.541$ & $4.181 \pm 0.476$ & $4.021 \pm 0.573$ & $3.986 \pm 0.554$ & $4.170 \pm 0.537$ \\
\hline $\begin{array}{l}\text { College English } \\
\text { Level } 4 \text { (272) }\end{array}$ & $4.138 \pm 0.497$ & $3.806 \pm 0.621$ & $4.026 \pm 0.478$ & $4.203 \pm 0.468$ & $4.063 \pm 0.506$ & $4.082 \pm 0.531$ & $4.219 \pm 0.497$ \\
\hline $\begin{array}{l}\text { College English } \\
\text { Level } 6 \text { (61) }\end{array}$ & $4.154 \pm 0.428$ & $3.899 \pm 0.621$ & $4.061 \pm 0.463$ & $4.242 \pm 0.298$ & $4.072 \pm 0.462$ & $4.051 \pm 0.448$ & $4.189 \pm 0.526$ \\
\hline$F$ & 0.25 & 0.74 & 0.95 & 0.62 & 0.74 & 3.25 & 0.86 \\
\hline$P$ & $<0.05^{\star}$ & $>0.05$ & $>0.05$ & $>0.05$ & $>0.05$ & $<0.05^{\star}$ & $>0.05$ \\
\hline \multicolumn{8}{|c|}{ Learning secondary foreign languages } \\
\hline Yes (73) & $3.973 \pm 0.456$ & $3.753 \pm 0.527$ & $3.967 \pm 0.517$ & $4.118 \pm 0.465$ & $3.975 \pm 0.440$ & $4.010 \pm 0.511$ & $4.146 \pm 0.502$ \\
\hline No (1008) & $4.137 \pm 0.476$ & $3.810 \pm 0.630$ & $4.004 \pm 0.522$ & $4.195 \pm 0.470$ & $4.039 \pm 0.557$ & $4.014 \pm 0.547$ & $4.186 \pm 0.528$ \\
\hline$T$ & 2.86 & 0.74 & 0.58 & 1.35 & 0.96 & 0.06 & 0.63 \\
\hline$P$ & $<0.05^{\star}$ & $>0.05$ & $>0.05$ & $>0.05$ & $>0.05$ & $>0.05$ & $>0.05$ \\
\hline \multicolumn{8}{|l|}{ Studying overseas } \\
\hline Yes (46) & $4.270 \pm 0.555$ & $3.848 \pm 0.703$ & $4.040 \pm 0.594$ & $4.283 \pm 0.445$ & $4.080 \pm 0.554$ & $4.098 \pm 0.534$ & $4.159 \pm 0.557$ \\
\hline No (1036) & $4.120 \pm 0.472$ & $3.804 \pm 0.620$ & $4.000 \pm 0.518$ & $4.186 \pm 0.471$ & $4.032 \pm 0.551$ & $4.010 \pm 0.545$ & $4.185 \pm 0.525$ \\
\hline$T$ & -2.10 & -0.47 & -0.50 & -1.36 & -0.57 & -1.07 & -0.32 \\
\hline$P$ & $<0.05^{\star}$ & $>0.05$ & $>0.05$ & $>0.05$ & $>0.05$ & $>0.05$ & $>0.05$ \\
\hline
\end{tabular}

${ }^{*} P<0.05$.

Table 2. Cultural competence scores by variables $(M \pm S D)$.

about. In daily communication, most nurses also knew how to respond to patients. Command of the cultural encounter increased with age. Thus, when senior nurses taught nursing procedures to young nurses, the young nurses learned nursing knowledge, nursing practice, and also cultural encounters.

\subsubsection{Cultural knowledge had the lowest score}

Cultural knowledge had an abundance of content, which included not only basic nursing knowledge and specialized knowledge but also customs, taboos, politics, economics, religions, diets, social etiquettes, insurance-related knowledge, language, and dialects. In China, nursing universities and colleges, and even nursing training programs, focus little attention on cultural-related knowledge, and nurses do not fully realize the importance of culture-related knowledge. Therefore, universities and hospitals should add culture-related courses into training programs, regularly update the courses, and permit nurses to keep up with the times and be aware of the newest information and knowledge. 


\subsection{Demographic factors influencing cultural competence among registered nurses}

\subsubsection{Age}

Age was related to cultural consciousness. Nurses $>40$ years of age had the highest score among all nurses $(P<0.05)$ because older nurses tended to be more sophisticated and experienced, and they more easily realized, understood, and accepted cultural differences. Hospital training courses should take the nurse's age and educational background into account or invite senior nurses to share or demonstrate their nursing experience with young nurses.

\subsubsection{Level of hospital care}

The level of hospital care had a significant difference in the dimensions of cultural consciousness, cultural knowledge, cultural skills, cultural desire, and cultural experience $(P<0.05)$. Nurses from secondary and tertiary hospitals scored higher than nurses from community hospitals. Thus, the level of hospital care and hospital size had a direct impact on a nurse's cultural consciousness, cultural knowledge, cultural skills, cultural desire, and cultural experience. Hospitals have their own history, conditions, and modes of operation. Hospitals that deliver a higher level of care tended to emphasize multidisciplinary development, interdisciplinary collaboration, and international communication, and nurses had opportunities to open their minds and participate in academic communication; thus, the academic level increased at the same time. At higher-level hospitals, nurses were more likely to meet patients with different cultural backgrounds and medical staff from different medical organizations. The more experience the nurses had, the more was the cultural competence that accumulated.

\subsubsection{Level of English mastery}

Nurses with high levels of English mastery had significant differences in the dimensions of cultural knowledge, cultural encounters, and cultural desire $(P<0.05)$. Nurses who were proficient in English could communicate well with foreign patients, understood the patients' needs, and easily accepted cultural differences. Currently, many Chinese hospitals have organized foreign language classes, such as English and Japanese courses, to increase the interests of nurses. Hospitals, especially tertiary hospitals, provide opportunities to study abroad in an effort to spur nurses to learn foreign languages. There have been many 1-year baccalaureate programs in nursing science that have been launched in China.
A number of programs in the US, Australia, Singapore, Finland, and the UK have been set up to help nurses obtain advanced nursing experiences, as well as to increase interest in studying.

\subsubsection{Learning secondary foreign languages}

Nurses who were learning or had learned secondary foreign languages scored high in the dimension of cultural consciousness $(P<0.05)$. Learning secondary foreign languages is an indicator that nurses are willing to learn more, and in the process of studying, they learned not only languages but also culture, customs, knowledge, history, and humanistic background.

\subsubsection{Studying overseas}

Nurses who had overseas studying or training experience scored high in the dimension of cultural consciousness $(P<0.05)$. When nurses studied, or trained, overseas, they became involved in the local life, were directly and deeply touched by the different cultures, and thus knew more about culture-related knowledge.

\section{Implications for nursing and policy}

There are various definitions of cultural competence, and the most popular one is the concept of possessing the attitude, knowledge, and skill necessary for providing quality care to a diverse population (i.e., the capacity to deliver culturally appropriate care). ${ }^{10}$ Owing to the recent escalating growth in global migration, and the consequent changes in emotions and lifestyle, the concern related to culturally competent health care has increased dramatically. In China, many hospitals have gradually focused attention on the cultivation of cultural competence among nurses. In this study, cultural competence consisted of seven dimensions (cultural awareness, cultural practice, cultural encounter, cultural desire, cultural skill, cultural experience, and cultural knowledge). There were many factors contributing to the cultivation of cultural competence among nurses, such as age, level of hospital care, level of English mastery, learning secondary foreign languages, and studying overseas. Therefore, numerous measures could be undertaken to increase the cultural competence of nurses. First, the Department of Education should attach importance to the cultivation of cultural competence among nurses and include cultural competence as part of the regular curriculum. Second, examinations for the certification of registered 
nurses should include specialized skills and knowledge, as well as cultural competence courses. Third, cultural competence courses should also be included in the probationary nurse training programs. Finally, when we set up cultural competence training courses, we could absorb overseas training experience, in addition to considering the actual conditions in China. The cultural competence courses in hospitals should consider the individual differences among nurses and their professional competence levels. Various training models should be undertaken to increase interest among nurses. Indeed, the journey to increase the overall level of cultural competence among nurses is lengthy.

\section{Conclusions}

Patients will benefit from health-care providers who value the patients' context and acknowledge their way of life in the provision of treatment plans and care..$^{28,29}$ Health centers should continue to take cultural competencies into account and take actions to bridge the current gaps between nurses, physicians, and patients. Institutions should also be aware of the importance of carrying out cultural competence programs with respect to cultural competence training. In this study, we found that the overall level of cultural competence among registered nurses in Shanghai's Pudong New Area was moderate. Among the seven dimensions, cultural encounter had the highest score and cultural knowledge had the lowest scores. Furthermore, age, level of hospital care, mastery over a secondary language, mastery of English level, and studying overseas were the influencing factors. Therefore, we can promote nurses' cultural competence according to the nurses' age, hospital level, and language level. Cultural competence-related courses should be increased, and various forms of training should be undertaken to enhance the interest of nurses. In this study, we find that there is still room for improvement of cultural competency among registered nurses in Shanghai. Cultural competence is not easy to understand and measure, and there will be a long journey ahead for nurse researchers and nurse educators to make all nurses become culturally competent. The challenge still exists to assess cultural competence

\section{References}

1. Casillas A, Paroz S, Green AR, et al. Cultural competency of health-care providers in a Swiss University Hospital: self-assessed cross-cultural skillfulness in a cross-sectional study. BMC Med Educ. 2014;14:19. among nurses in clinical practice and its effect on patient outcomes. ${ }^{4}$

\section{Limitations}

The data in this study were obtained from surveying registered nurses in Pudong New Area in Shanghai, which limited the generalization of the findings. Because Shanghai is a municipality directly under the Central Government, the registered nurses of Shanghai may have differences in cultural competence relative to nurses of other regions. Further research is warranted with larger samples to ensure meaningful representation of nurses from other geographic regions.

\section{Acknowledgments}

All authors are in agreement with the contents of the manuscript. The authors are grateful to all participants for their involvement in the study. The interviews were organized with the help of an independent person who was not the coauthor of this paper. She helped to enter the survey data into the computer to ensure the accuracy.

The authors would also like to thank the National Natural Science Foundation of China for funding the study through a grant (No. 71473178).

\section{Ethical approval}

The study was approved by the hospital Ethics Committee of Shanghai East Hospital, Shanghai, China (No. 2014-053). Approval for conducting the study was obtained from the head nurses of the surveyed units and the Director of the Nursing Department of the surveyed hospitals. The study objectives and the method were explained to each participant in detail. Participation by all the participants was ensured to be voluntary. Anonymity and confidentiality were ensured. The survey was conducted after consent was received from each participant.

\section{Conflicts of interest}

All contributing authors declare no conflicts of interest.
2. Betancourt JR. Cross-cultural medical education: conceptual approaches and frameworks for evaluation. Acad Med. 2003;78:560-569.

3. Brach C, Fraser I. Can cultural competency reduce racial and ethnic health disparities? 
A review and conceptual model. Med Care Res Rev. 2000;57(suppl 1):181-217.

4. Loftin C, Hartin V, Branson M, Reyes H. Measures of cultural competence in nurses: an integrative review. Sci World J. 2013;2013:289101.

5. Betancourt JR, Green AR, Carrillo JE, AnanehFirempong O. Defining cultural competence: a practical framework for addressing racial/ethnic disparities in health and health care. Public Health Rep. 2003;118:293-302.

6. Beach MC, Price EG, Gary TL, et al. Cultural competency: a systematic review of health care provider educational interventions. Med Care. 2005;43:356-373.

7. Beach MC, Gary TL, Price EG, et al. Improving health care quality for racial/ethnic minorities: a systematic review of the best evidence regarding provider and organization interventions. BMC Public Health. 2006;6:104.

8. Campinha-Bacote J. Inventory for Assessing the Process of Cultural Competence among Healthcare Professionals-Revised (IAPCC-R). 4th ed. Cincinnati: Trans cultural Care Associates; 2003.

9. Robins LS, Fantone JC, Hermann J, Alexander GL, Zweifler AJ. Improving cultural awareness and sensitivity training in medical school. Acad Med. 1998;73(suppl 10):S31-S34.

10. California Endowment. Principles and recommended standards for cultural competence education of health care professionals. 2003. Woodland Hills, CA: The California Endowment; 2003.

11. Saha S, Beach MC, Cooper LA. Patient centeredness, cultural competence and healthcare quality. J Natl Med Assoc. 2008;100:1275-1285.

12. Betancourt JR, Green AR. Commentary: linking cultural competence training to improved health outcomes: perspectives from the field. Acad Med. 2010;85:583-585.

13. Giger J, Davidhizar RE, Purnell L, et al. American Academy of Nursing Expert Panel Report: developing cultural competence to eliminate health disparities in ethnic minorities and other vulnerable populations. J Transcult Nurs. 2007;18:95-102.

14. Jeffreys MR. Teaching Cultural Competence in Nursing and Health Care. 2nd edition. New York, USA: Springer; 2010.

15. Seeleman C, Hermans J, Lamkaddem M, Suurmond J, Stronks K, Essink-Bot ML. A students' survey of cultural competence as a basis for identifying gaps in the medical curriculum. BMC Med Educ. $2014 ; 14: 216$.
16. Helen $\mathrm{R}$, Lance $\mathrm{H}$, Deborah D. A comparative analysis of cultural competence in beginning and graduating nursing students. ISRN Nurs. 2013; 2013:929764.

17. Ma LL, Peng YQ, Jiang $H$. Construction of cultural competence scale for clinical nurses. Chin J Modern Nurs. 2015;21:3479-3484 (in Chinese).

18. Sherwood G, Liu H. International collaboration for developing graduate education in China. Nurs Outlook. 2005;53:15-20.

19. Li XD, Acorn S. Nursing administration in China. J Nurs Adm. 1996;26:12-13.

20. Yun $H$, Shen J, Jiang A. Nursing shortage in China: state, causes, and strategy. Nurs Outlook. 2010;58:122-128.

21. Ministry of Health of People's Republic of China. High Quality Nursing Care Document. 2011. http://www.nhc.gov.cn/xxgk/pages/viewdocument. jsp? dispatchDate=\&staticUrl=/zwgkzt/wsbysj/ 201103/51078.shtml \&indexNum=000013610/201103744\&manuscriptld=51078. Accessed March 25, 2019 (in Chinese).

22. McCabe DE, Alvare CD, McNulty SR, Fitzpatrick JJ. Perceptions of physical restraints use in the elderly among registered nurses and nurse assistants in a single acute care hospital. Geriatr Nurs. 2011;32:39-45.

23. Hurlock-Chorostecki C, Kielb C. Knot-So-Fast: a learning plan to minimize patient restraint in a critical care. Dynamics. 2006;17:12-18.

24. Du DD, Chen JL, Huang WQ. Survey of the demands for knowledge of humanistic and cultural competence in college nursing students. J Nurs Sci. 2007;22:59-60 (in Chinese).

25. Ge YY, Gong XS. Study on the cultural sensitivity measurement for nursing undergraduate students. Chin J Behav Med Sci. 2008;17:953-955 (in Chinese).

26. She P, Zhang J. Survey of status quo and influencing factors of cultural competence of clinical nurses in Wuhan city. Chin Nurs Res. 2013;27:2335-2338 (in Chinese).

27. Huo M. The development of rating scale of cultural competence and investigation on the status of cultural competence of nurses. China Med Univ. 2009 (in Chinese).

28. Domenig D. Migration, Drogen, transkulturel leKompetenz. Bern: Hans Huber Verlag; 2001.

29. Domenig D. Professionalle transkulturelle Pflege. Bern: Hans Huber Verlag; 2001. 